\title{
Road map for implementing AI-driven Oulu Smart excavator
}

\author{
Hassan Mehmood ${ }^{1}$, Mikko Hiltunen ${ }^{2}$, Tomi Makkonen ${ }^{2}$, Matti Immonen², Susanna \\ Pirttikangas ${ }^{1}$ and Rauno Heikkilä ${ }^{2}$ \\ ${ }^{1}$ Center for Ubiquitous Computing, University of Oulu, Finland \\ ${ }^{2}$ Construction Automation Research Center in Civil Engineering, University of Oulu, Finland
}

firstname.lastname@oulu.fi

\begin{abstract}
-
Machine control systems are advancing side by side with the adoption of 5G and growing trends of the internet of things (IoT) has made autonomous excavators more ubiquitous. The autonomous excavators have gained significant interest in the earthworks area, due to their enhanced productivity for long hours, safety, space exploration, mining and construction work. However, A great amount of effort is required to address many existing challenges such as adaptive movement and control, task planning (digging, moving debris, etc.), collaborative work with other machines and humans. In this study, we review the state of the art and provide artificial intelligence (AI-) driven road map for implementing a complete autonomous framework for the earth-moving machine to our test platform 'Smart Excavator'. Furthermore, the challenges and required effort to implement the framework are also discussed in comparison with existing literature.
\end{abstract}

\section{Keywords -}

construction engineering;mining;excavation;smart excavation;artificial intelligence; data collection; machine learning; excavator

\section{Introduction}

The automated and autonomous excavators can be seen as a logical outcome, or at least enabled by, engineering mega-trends namely AI, automation and, IoT, and Space exploration. In one sense we can classify an excavator as a manipulator that is working on the field, using IoT and 3D models and control hydraulics for digging; however, if this would be the case self-moving excavators would already be plenty in the field. What is lacking from this is a simplified view of the complexity of the always-changing work environment where the excavator needs to smoothly operate. All this still while maintaining safety and fleet capabilities with machines and humans.

Makkonen et al. suggested that road designer's design models (3D surface) can be used as a source for path generation for excavators [1]. High usage of machine control systems in earth-moving machinery gives effortless access to target 3D surfaces, real-time localization, and kinematic positioning of the excavator. Given that this kind of excavator is also part of IoT, that makes the autonomous excavator is already capable of updating the information about its activities and processes to the network.

The operational performance of excavators is continuously being improved. As it holds a great potential market for construction engineering area, where earth-moving machines require planning and precise control [2] to execute various tasks from trenching to larger-scale operations. For example, founding and constructing the supporting layers of the highway. The effective operation time of the excavators in construction is maximized in the majority of cases to cut down the cost of building, which holds a potential challenge and opportunity for automation-based excavators.

The automation level for excavators have been categorized into various types, the most common are, i) humancontrolled machines, ii) remote-controlled machines, iii) semi-autonomous machines, iv) fully-autonomous machines [3, 2]. . The automation and autonomy traits in excavators can however be increased, considering the ongoing challenges in construction engineering and earth moving machines area. Apart from the heterogeneous and circumstance-dependent characteristics of the terrain, ensuring the safety and efficiency of these machines is a great challenge toward fully autonomous excavators [3].

In this study, we propose a framework to implement AI-driven autonomous excavators using the data streams from the excavator itself in real-time. The study is one of the first attempts, to consider both edge and cloud-driven challenges and solutions for implementing an autonomous excavator system. Considering, the low latency and realtime processing challenges, a hybrid approach is proposed, see Section 4. First, we identify the requirements to implement AI-driven autonomous framework from existing literature, following that, a framework is proposed. In addition, the system will be capable of handling drifting scenarios and the occurrence of concept drift while modeling, which can affect the learning of models and decision-making.

The rest of the paper is structured as Section 2 details 
the state of the art, Section 3 lists the requirements to implement the autonomous framework. Section 4 describes the framework and Section 5 presents a use case for the framework. Finally, we discuss the limitations and concrete challenges along with the conclusion.

\section{Literature Review}

\subsection{Methods for autonomous excavators}

In previous years, various toolboxes and methods were introduced for autonomous excavators. Back in 1998, the Robotic Institute at Carnegie Mellon University, USA, created an excavator model capable of finding and filling up the intended truck without any human interaction [4]. Similarly, Lancaster University's intelligent excavator (LUCIE) [5] demonstrated several automated robotics features in the excavation process e.g. controlling motion of the bucket. Unlike traditional excavators, autonomous excavators now have become perceptive, as they are equipped with cutting edge sensors, cameras, a global positioning system (GPS), lidar, radar, and laser beams mostly [3, 6]. Such equipment enhances the autonomy of the excavators, as they can collect raw data, and analyze the data using machine learning to develop an understanding of the designated environment.

A study by [6], proposed a real-time multi-frame Long Short-Term Memory (LSTM-) based method with a You Only Look Once (YOLO-)v2, as the main backbone to accumulate useful information from image frames for more robust image detection, with decreased computational effort. The developed approach resulted in near human-level accuracy to detect different types of objects under varying light conditions, lots of dust, etc. However, the performance of the method decreased in low light conditions.

Another study by [7], focused on the soil excavation operation of autonomous excavators, to maximize the performance of the digging process like an expert human. With the varying soil dynamics, capturing soil characteristics by adaptive models can be challenging. Dynamic Movement Primitives (DMP), was adopted to tackle the complex soil dynamics. In particular, DMP uses virtual dynamics approach to structure and preserve the true trajectory shape to the finishing point i.e. transition to move and dump excavated material [7]. The implemented algorithm effectively learns the best position trajectories, ensuring excessive adaptation to changing soil properties with additional safety and robustness.

There are different ongoing challenges to operate excavators autonomously such as, variable environmental conditions, weather, changing lighting conditions, dropping piles of materials to a place or truck, digging of the material with its natural integrity, and avoiding collisions with the objects in construction site [8]. In this regard, a new architecture and algorithms were proposed by [8] for autonomous excavators. The system was built by a central perception module, integrating perception, planning, and control. The perception module fuses the information from equipped 3D-Lidar and camera outputs, to perceive 3D models of material, dumping site, obstacles, and others. The system was also capable of handling sudden changes in the environment to provide safety e.g. movement of animals on construction sites.

\subsection{Applications of autonomous excavator}

So far automation is used to streamline the earthworks on infrastructure and construction sites in most commercial solutions [9, 10, 11] using GNSS localization and boom-mounted IMU sensory to pinpoint the bucket or blade of the machinery. This allows the operator to measure grades and slopes with the machine itself and removes the need for laborious marking the site with physical signs as the operator can view the plans besides the machine from the system monitor. Now gaining popularity are also cloud-based management services like [12] which are already widely in use on Finnish public construction sites to manage the full project. Cloud services enable flexibility as any changes made and uploaded to design models or other information are immediately available to everyone involved including the excavator operators. It also helps to reduce material and fuel expenses as well as labor costs and machine hours as the service eases machine management on a whole scale.

The machine manufacturers [9] and automation system providers [10, 11] are also introduced grade control to semi-automatically excavate grades and slopes with improved precision. The operator controls the joystick and automation keeps the bucket on grade. Semi automation controls also include depth control for preventing overcutting. A study by [10], have also showcased safety functions to limit the working area by virtual walls to prevent collisions, when working on tight construction areas and weighting system for the bucket to have better material management on truck loading in construction or for example in gravel pits.

Japan institute [13] stated that automatic and unmanned construction machines could be used to sort debris in reconstruction sites to remove or restore broken roads or structures or demolish or rebuilt damaged houses in places that cannot be safely accessed by humans after disasters. Also, automation could be used in highly repetitive job sites in construction on tasks like truck loading to let human operators focus on more challenging tasks.

The Space programs [14] introduced plans to send unmanned construction machinery to explore the surface and build lunar and Mars stations before sending manned flights. Automatic excavation of possibly unknown soil 
materials is a crucial task to establish any buildings and efficient usage of the local materials would greatly reduce the payload of the flights.

\section{Requirements for $\mathrm{AI}$ driven autonomous excavators}

\subsection{Intelligent Control}

An autonomous excavator requires adaptive and robust control procedures to be able to operate with full control along with an understanding of its surroundings that is in constant change. The remote-controlled excavators in past have used feedback linearization technique, impedance controller, non-linear proportional-integral controlled for optimized interaction.

Usually, for self-operation, the source of movement is hydraulic power, but lately, electric machinery like [15] are on the rise. The base excavator can move and rotate reasonably freely on the work area using tracks or all-wheel drive. The end-effector also has 4 degrees of freedom as the cabin rotates and the boom, arm, and the coupler for the bucket are all linked together with a rotational axis and have actuators to manipulate them. When an excavator is used as a multipurpose tool a rototill adds versatility by adding 2 more DOFs for the end-effector. To fulfill the requirement for control of its movements, all kinematics links from the base to end-effector should be measured and the movements performed in a precise and accurate method enough.

Understanding the surroundings is mandatory for autonomous machines in general and can even be the key difference between AI-controlled machines and normal machines as humans have limited situational awareness and cognitive load. Typical sensor solutions for this are $2 \mathrm{D}$ cameras with various sensor types like RGB, heat, and multi-spectral; for scanning and modeling the environment 3D stereo cameras and lidars are typically used. For localization on the work site GNSS-location systems are most used, with two antennas they provide heading and angle measurements in addition to location data. In larger job sites, local fixed reference stations provide correction signals to the localization to achieve centimeter-level preciseness.

Specific to autonomous construction work machines like an excavator, a dynamic site-wide plan to follow is provided and AI needs to be able to full fill the plan. Typically this plan comes from humans like road designers, but there is no reason that this plan is also from $\mathrm{AI}$ in the future. The plans are typically presented in CAD models, but the surge of structured digital information in the forms of building information modeling (BIM) is fast gaining ground also outside the Nordic countries.

\subsection{Motion Planning}

The earth moving machines in general for earth cutting and standard movements require force feedback to sense the surrounding environment especially the processed soil. Force control also would help to have more advanced loading and other movements as skilled human operators also use wide haptic feedback from the machine. The force feedback could be also used in tandem with vision sensors in events, where continuous movement and digging is performed with a high chance of collision with static objects in excavation site e.g. collision with another machine, falling to a ditch or a dump, hitting a tree or a rock, or others. The force feedback in excavation sites can be provided using sensor-based data collection adjoined with real-time sensing and AI-driven learning methods.

Several scientific studies have tried to address the challenges of nominal motion planning in excavation sites. A study by [4] proposed an autonomous loading system using a perception engineering-based approach, using laser scan to detect truck model and soil without change of texture. Similarly, a study by [16] introduced a framework for each work machine to minimize human involvement in the excavation site. In addition, different approaches and algorithms have been experimented to optimize the process of planning and perception for earth-moving machines.

\subsection{Simulation Environment}

The major reason to use simulation and virtual environments is safety and real-time tests, mapping, and learning in continuously changing environments. In a study by [17], excavator Menzi Muck M545 simulated the excavation process using a planning and control approach. The excavator was able to reliably fill buckets using force trajectory-based dig cycles. [17]. In another study, a co-simulation framework was developed based on MATLAB-SIMULINK for modeling controller and OPNET to simulate communication network and optimize the remotely controllable excavator [18]. Simulation-based methods are also prominent to understand the landscapes and changes in the environment using sensor data [2].

The simulation environments integrated with the complete autonomous excavation system are useful to demonstrate the fieldwork virtually, along with planning and monitoring. As the earth moving machines are equipped with sensors like IMUs, a global positioning system (GPS), pressure sensors, and possible weather sensors, and others. The sensor data can be utilized to develop perceptionbased autonomous excavation systems using artificial intelligence approaches. Therefore, developing and integrating simulation tools or environments with an autonomous excavation framework can help in planning and monitoring data in real-time. Lastly, the simulation environment can 
be effective for monitoring the sensor feeds continuously for detecting the possible drifts in streaming data [19].

\subsection{Tools and Frameworks}

Construction work-specific autonomous excavation machines require dynamic technology solutions to adapt to diverse characteristics of soil and building materials, accompanied by a coherent modeling plan. Typically, this plan comes from expert individuals in the respective field such as road designers, civil engineers, and urban planners. Therefore, it should be possible that future AI-driven intelligent solutions could learn from historical plans and construction sites, and do minor adjustment to the construction plans such as mass swap, layer thicknesses with minimal or no human intervention. In this regard, the autonomous earthwork machines require dynamic and light computational tools and frameworks, which can be used in both cloud and edge architectures for effective excavation solutions.

The excavation plans are typically presented in CAD models, but the surge of structured digital information in the forms of building information modeling (BIM) is fast gaining ground both in Nordic countries and outside as well. The equipped sensors with earth machines are configured using CAN bus and other platforms. The sensed feeds from sensors are often further pre-processed and explored using MATLAB/Simulink, standard python libraries, and different simulation tools. With cloud and edge-based platforms low latency computing and analysis tools are also gaining prominence for generating insights e.g. at cloud end Apache Spark, Apache Kafka, Kibana, and others [20]. However, as with increasing trends of IoT and implementation of 5G, the earthwork machines would need more edge computing capabilities. Therefore, light computational tools and frameworks with the ability to handle low latency data streams are needed.

\section{Theoretical Framework}

\subsection{Framework of AI driven Autonomous Excavator}

The proposed framework will be implemented on hybrid cloud and edge architecture, considering the ongoing trends of IoT and the implementation of 5G. In this regard, we propose an abstraction level hybrid architecture that constitutes both edge and cloud-based solution requirements, please see Figure 2 and Section 4.2.

The first block of the AI-driven framework is "Sensing and Planning", which is designed based on the idea of sensing and adaptive learning in real-time, please see Figure 1 . It first utilizes the sensors to make model adjustments in both local and global aspects. Then, the tasks are created by the task planner based on the work maps and building information models. The data from sensors such as laser

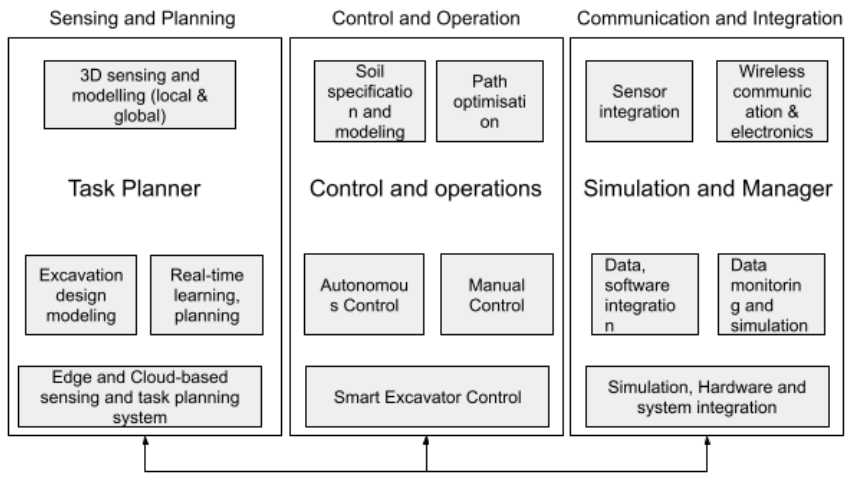

Figure 1. Framework for AI-driven Autonomous Excavator, adapted from [21]

sensors and 3D Lidar sensors will be used for developing adaptive learning methods for intelligent movement. In addition, as data from sensors is continuously changing and real-time in nature, therefore, due to changes in statistical properties of data, a concept drift can occur [19]. The drift is often due to the malfunction of the sensor or actuator. Such changes in the data stream can result in ineffective predictions from learners e.g. less efficient object detection for collision-free movement. Therefore, concept drift detection and adaption methods are also a key requirement for learners operational in real-world settings.

The second block "Control and Operation" in the framework incorporates and implements the sensed feeds and knowledge from learners to perform excavation operations in a real environment. In this regard, the requirements described in Section 3. to adapt and characterize the dynamic nature of excavation terrain is handled by using the control procedures from implemented models. Furthermore, collision is possible on excavation sites due to animal movement, object movement, truck movement, and debris., which requires collision detection and path planning methods. All previously mentioned procedures are controlled and managed in this layer. Additionally, the likelihood of device and machine failures is possible, which can make the autonomous operation by excavator impossible. Therefore, a hybrid approach is implemented for controlling the excavator i.e. in case of failure or system shutdown, an administrator on site can flexibly take over the machine. For such cases, an alarm system will be implemented using the sensed feeds from the machine that can be enabled to receive alerts and view the performance of the machine using a tablet or other mobile device, please see Figure 2

The third block "Communication and Integration" constitutes control and monitoring tasks during the excavation operations at a respective site. We propose to integrate and continuous monitoring and simulation system with the pre- 
vious two mentioned technology stacks. Keeping in view the new methods of communication aided by $5 \mathrm{G}$ support and simulating sensor feeds can make the operations of excavators more efficient. Additionally, the integration of developed intelligent packages for adaptive control and movement of excavators is executed in this block. This creates the possibility to monitor and simulate the operations before going to the excavation site, as well as allows continuous monitoring facility during real-world operations.

\subsection{Edge AI architecture for Autonomous Excavator}

As the world is rapidly moving towards IoT and advance communication paradigms. With the increased number of interconnected physical devices, developments are being made in edge computing to handle the sheer amount of data being shared. There are several application areas from edge computing assisted IoT, which includes household IoT, industrial IoT, autonomous vehicles, and $5 \mathrm{G}$ networks [22].

In industrial IoT, there are several opportunities available which include the capability to continuously monitor production lines in real-time using sensing feeds from a multitude of sensors [22]. For example, the data produced from equipped vibration sensors from an excavator's pump can help in distinguishing safety levels using mechanical vibrations. Similarly, the multitude of sensors can also be used for collision-free movement, identifying the position of excavators, and acquiring the movement routes of other vehicles in the vicinity.

The overall approach to implement AI-driven autonomous excavators using edge computing approach is illustrated in Figure 2. The excavator machine is equipped with a myriad of sensors that are continuously producing and pushing data to the database at cloud and edge. The data stored in the cloud is further used to simulate and generates insights to better monitor and control the excavators in exceptional situations. Additionally, the data at cloud is used to model and train learners for adaptive control and collision-free movement, as described in Figure 1. The sensed data can however further be utilized to ensure security and for prompt administrative response in case of malfunctioning or hazardous situations. For example, if the autonomous module of the excavator goes offline and at the excavation site their exceptional circumstances (falling of debris or other vehicular movements etc), before going offline the system will send an alert to the mobile or tablet device of the administrator, and the excavator can be switched to manual mode. Following that, the learned knowledge is then transferred to an edge network that stays persistent with excavators, reducing the latency issues. However, if a concept drift occurs in drawn predictions, then the new information is pushed at the edge from the cloud to retrain the learners. The architecture is further extended by providing command and control using smart devices such as mobile phones and tablet devices.

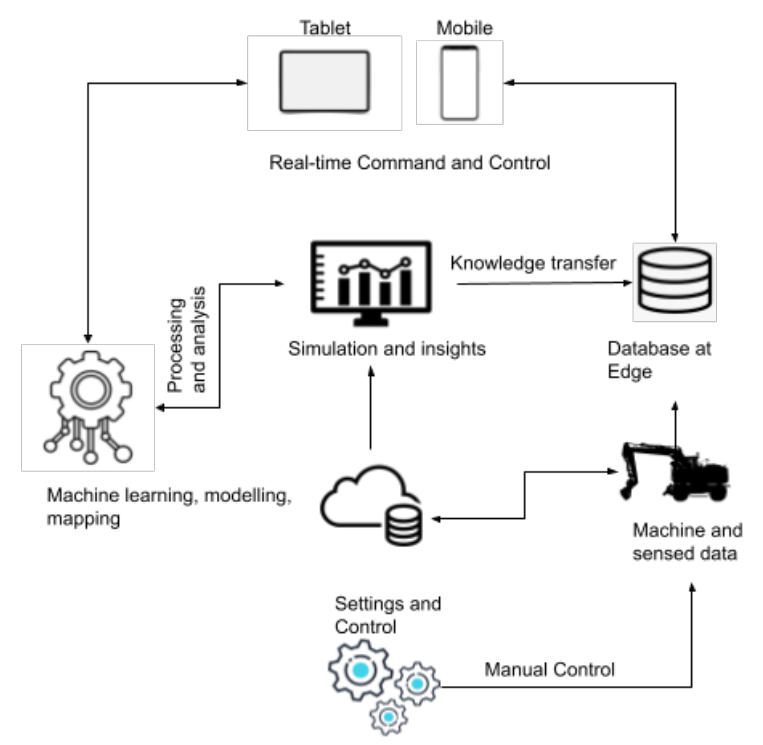

Figure 2. Basic Edge AI driven architecture for autonomous excavator

\section{Use Case}

In our developed model-based autonomous control, Machine Control Model (MCM) is used for the trajectory generation for the Smart Excavator. In these examples, the models are in LandXML file transfer format saved using Finnish Infra model standards. For adaptive development and testing purposes, the visual programming language Grasshopper included in Rhinoceros CAD software is used to model the digital twin of the Bobcat E85 Robotic Excavator and to load and partition the lines and triangulation from the Inframodel for the trajectory generation.

The use of Grasshopper allows easy high parametrization of the trajectories. The software includes various ready function blocks from basic calculations to graphics computation. Also, own custom blocks can be written with custom expression or C sharp script, python script, or VB script. The visual preview of the digital twin in Rhinoceros is useful for examining the trajectories for errors before testing them with the actual robotic excavator. A major drawback is that there seems not to be a quick workaround to convert created grasshopper code to the standalone program to the Smart Excavators control system since code often contains blocks with various scripting languages. The Grasshopper itself is rather dependent on Rhinoceros because its core product uses many geometry functionalities provided by the CAD software. 
The design lines from the Machine Control Models are used to define the working area and to compute cartesianspace paths to cut the model surface. Adjacent and parallel lines are filtered and when necessary, divided according to working region. The bucket paths are then computed between those lines to excavate the designed surface. We also use the lines from the model to create surfaces with Rhino's loft tool instead of using the triangulated surface from the model to avoid imprecision and confusion in the edges of the triangles. Alternatively, para-metrically controlled Bezier curve-shaped shoveling paths are created in grasshopper and the endpoints are linked to soil surface for mass excavations.

To define the trajectories, the path's curve or line is divided into segments which are then evaluated with the associated surface. The evaluation gives a surface normal vector for each segment. Every path point is translated to the excavator's local coordinates. The formed frame for each path segment is yet compared to the excavator's mainframe to compute Euler angles. The path is then converted to joint space using inverse kinematics. After that, with information from the surrounding environment and cooperating vehicles such as a dumpster, additional joint space movements are generated to complete the workflow and to dump the excavated material to the defined location. For example, onto a truck bed.

The trajectories are tested and analyzed with the 8,5ton Bobcat Smart excavator. The excavator has retrofitted electric valves to control the hydraulics. An on-board PC is in charge of controlling the valves and forwarding signals from joysticks and other controls and data from boom IMU sensors. It is connected to an industrial PC with a CAN bus over a local $5 \mathrm{GHz}$ wireless network. The industrial PC runs Matlab/Simulink controller program which has a separate controller for each actuator with online adjustable tuning. The controller has three modes: remote control, teach-in to record and repeat workflow, and automatic control for executing the model-based trajectories. So far the automation tests have included different special-shaped surfaces like stairs and various slopes to iterate and tune the trajectories and controllers to better precision. Experiments to compare excavation aboard, remote controlling, and automation have been also planned.

\section{Discussion}

Artificial intelligence has been identified as a driver for sustainable development [24]. Within the autonomous vehicle community and industrial IoT, the main considerations of sustainability are resource optimization and safety [24]. Intelligent automation saves resources when optimal real-time planning can be made through intelligent algorithms for enhanced fuel efficiency, leading to fewer emissions. We can recognize different layers of planning:

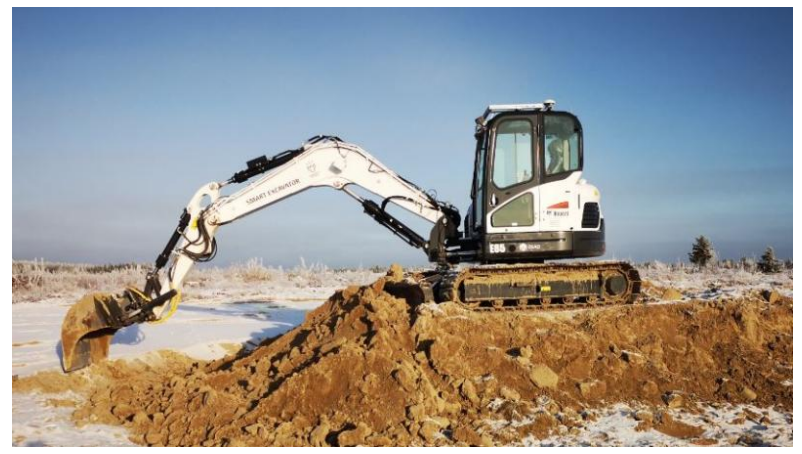

Figure 3. Smart excavation in progress at test site in winter conditions [23]

1) construction site planning 2) fleet intelligence 3) individual vehicles. The construction site planning could optimize material movements on-site to maximize material re-usage and minimize unnecessary loading, transport, and other material manipulation. Connected vehicles operating in fleets can optimize their cooperation to maximize activity and reduce wait time and overall work duration. For example, excavators and front loader can synchronize their operations to optimize piling or truck loading.

Automated monitoring of vehicles can be used in improving vehicle maintenance schedules. Environmental measurements like emission particles, temperature, and moisture can also be used to create climate-friendly earth moving machines by studying data generated from sensors and operational insights of existing excavators. Additionally, cloud computing and edge computing can play a pivotal role in produce climate-friendly and sustainable machines. As we know, the data generated from IoTs, and data from vehicular sensors becomes larger each day, therefore, generating insights and modeling the respective data can aid in achieving sustainable societal goals. In this study, we proposed a hybrid approach that includes both edge and cloud computing paradigms to retain historical information and help in quick real-time decision making for smart and autonomous excavators machines, please see Figure 2

The operation of individual vehicles can be optimized for efficient and energy prudent trajectories. Accuracy is to be improved where needed. Few centimeters of improvement on preciseness can save a lot of work and material on a bed of kilometers long highway but does small-scale landscaping need such accuracy? To be able to complete a complex planning task with multiple agents, task decomposition and task distribution need to be modeled. The edge architecture designed in this paper is designed to be used to leverage the high-volume data produced by these intelligent agents.

The construction machinery is increasingly equipped 
with various sensors to increase situational awareness of the operator. However, as mentioned earlier, presenting for example raw images from multiple different cameras could swiftly overwhelm the cognitive capacity of a human operator. Therefore handling of the sensor data should be somewhat automated and fused to form more clear and intuitive presentation using for example means of extended reality (XR). Also, adaptive AI control methods can be overwhelmed by too wide data streams. First and foremost irrelevant data or concept drift can slow down and even completely stall or mislead possible learning solutions [19].

The edge computing provides capabilities to access stored data, computing resources, and access with low latency, finding the closest available resources to the machines e.g. database at edge at the roadside unit, a database of an edge machine (car, truck, etc.) using a vehicle to vehicle communication [25]. Moreover, the infrastructure solutions which are used in edge computing can collect local resources efficiently in lesser time when compared with traditional computing paradigms. However, the concept itself is relatively new and has received prominent attention from both academia and industry as well as in transportation e.g. intelligent vehicles [25], therefore still requires exploration.

\section{Conclusion}

Industrial IoT has gained prominence in past decades, especially when it comes to construction engineering, the concept of "smart excavators", "intelligent excavation system", and "autonomous excavators" is on the rise in both academia and industry to produce industrial-grade earth moving machines. This article presents a approach to implement AI-driven intelligent framework for autonomous excavators after conducting a state-of-the-art review. Considering the ongoing advancements and challenges in ICT concerning autonomous and intelligent vehicles. A hybrid architecture is proposed along with three main technology blocks to implement AI-driven autonomous framework for smart excavation, Figure 1 and 2 .

The work presented in this study is still at its initial stages and shortcomings are expected. At the moment, the presented framework for AI-driven autonomous excavators has some limitations, e.g. the aspects to ensure the effectiveness of the system are not well covered, privacy and security concerns still require great effort. As with the implementation of $5 \mathrm{G}$ and new generation hardware, privacy and security constraints will also take a shift and will require advanced efforts.

In the future, we plan to move forward with development and present a fully implemented AI-driven autonomous framework with rigorous tests.

\section{References}

[1] Tomi Makkonen, Kelervo Nevala, and Rauno Heikkilä. A 3D model based control of an excavator. Automation in Construction, 15(5):571-577, 2006.

[2] Daniel Schmidt, Martin Proetzsch, and Karsten Berns. Simulation and control of an autonomous bucket excavator for landscaping tasks. In 2010 IEEE International Conference on Robotics and Automation, pages 5108-5113. IEEE, 2010.

[3] R Heikkilä, T Makkonen, I Niskanen, M Immonen, M Hiltunen, T Kolli, and P Tyni. Development of an earthmoving machinery autonomous excavator development platform. In ISARC. Proceedings of the International Symposium on Automation and Robotics in Construction, volume 36, pages 10051010. IAARC Publications, 2019.

[4] Anthony Stentz, John Bares, Sanjiv Singh, and Patrick Rowe. A robotic excavator for autonomous truck loading. Autonomous Robots, 7(2):175-186, 1999.

[5] David A Bradley and Derek W Seward. Developing real-time autonomous excavation-the lucie story. In Proceedings of 1995 34th IEEE Conference on Decision and Control, volume 3, pages 3028-3033. IEEE, 1995.

[6] Hooman Shariati, Anuar Yeraliyev, Burhan Terai, Shahram Tafazoli, and Mahdi Ramezani. Towards autonomous mining via intelligent excavators. In Proceedings of the IEEE/CVF Conference on Computer Vision and Pattern Recognition Workshops, pages 26-32, 2019.

[7] Bukun Son, ChangU Kim, Changmuk Kim, and Dongjun Lee. Expert-emulating excavation trajectory planning for autonomous robotic industrial excavator.

[8] Jinxin Zhao, Pinxin Long, Liyang Wang, Lingfeng Qian, Feixiang Lu, Xibin Song, Dinesh Manocha, and Liangjun Zhang. Aes: Autonomous excavator system for real-world and hazardous environments. arXiv preprint arXiv:2011.04848, 2020.

[9] Grade. URL https://wwW.cat.com/en_GB/ products/new/technology/grade.html.

[10] Automation for excavators, Oct 2020. URL https://novatron.fi/en/ automation-for-excavators/. 
[11] Machine control. URL https:// heavyindustry.trimble.com/en/products/ civil-construction/machine-control.

[12] Infrakit services. URL https://infrakit.com/ en/services/.

[13] Fujino Kenichi, Moteki Masaharu, Nishiyama Akihiko, and Yuta Shinichi. Towards autonomous excavation by hydraulic excavator - measurement and consideration on bucket posture and body stress in digging works. In IEEE Workshop on Advanced Robotics and its Social Impacts, 2013.

[14] Sanders Gerald, Larson William, and Sacksteder Kurt. Nasa lunar mining and construction activities and plans. 2009.

[15] Emobility. URL https://wwW.volvoce.com/ global/en/our-offer/emobility/

[16] Sung-Keun Kim and Jeffrey S Russell. Framework for an intelligent earthwork system: Part i. system architecture. Automation in Construction, 12(1):1$13,2003$.

[17] Dominic Jud, Gabriel Hottiger, Philipp Leemann, and Marco Hutter. Planning and control for autonomous excavation. IEEE Robotics and Automation Letters, 2(4):2151-2158, 2017.

[18] Hongnian Yu, Yang Liu, and Mohammad Shahidul Hasan. Review of modelling and remote control for excavators. International Journal of Advanced Mechatronic Systems, 2(1-2):68-80, 2010.

[19] Hassan Mehmood, Panos Kostakos, Marta Cortes, Theodoros Anagnostopoulos, Susanna Pirttikangas, and Ekaterina Gilman. Concept drift adaptation techniques in distributed environment for real-world data streams. Smart Cities, 4(1):349-371, 2021.

[20] Hassan Mehmood, Ekaterina Gilman, Marta Cortes, Panos Kostakos, Andrew Byrne, Katerina Valta, Stavros Tekes, and Jukka Riekki. Implementing big data lake for heterogeneous data sources. In 2019 ieee 35th international conference on data engineering workshops (icdew), pages 37-44. IEEE, 2019.

[21] Jongwon Seo, Seungsoo Lee, Jeonghwan Kim, and Sung-Keun Kim. Task planner design for an automated excavation system. Automation in Construction, 20(7):954-966, 2011.

[22] Jianbing Ni, Xiaodong Lin, and Xuemin Sherman Shen. Toward edge-assisted internet of things: From security and efficiency perspectives. IEEE Network, 33(2):50-57, 2019.
[23] Autonomously operating excavator developed at the university of oulu open application platform for further research and industrial cooperation. URL https://www.oulu.fi/university/news/ autonomously-operating-excavator-developed $\backslash$ -at-the-university-of-oulu.

[24] Ricardo Vinuesa, Hossein Azizpour, Iolanda Leite, Madeline Balaam, Virginia Dignum, Sami Domisch, Anna Felländer, Simone Daniela Langhans, Max Tegmark, and Francesco Fuso Nerini. The role of artificial intelligence in achieving the sustainable development goals. Nature communications, 11(1): $1-10,2020$.

[25] Jun Zhang and Khaled B Letaief. Mobile edge intelligence and computing for the internet of vehicles. Proceedings of the IEEE, 108(2):246-261, 2019.

[26] Jeonghwan Kim, Dong-eun Lee, and Jongwon Seo. Task planning strategy and path similarity analysis for an autonomous excavator. Automation in Construction, 112:103108, 2020.

[27] Heshan Fernando and Joshua Marshall. What lies beneath: Material classification for autonomous excavators using proprioceptive force sensing and machine learning. Automation in Construction, 119: 103374, 2020.

[28] David A Bradley and Derek W Seward. The development, control and operation of an autonomous robotic excavator. Journal of Intelligent and Robotic Systems, 21(1):73-97, 1998.

[29] David A Bradley, Derek W Seward, James E Mann, and Mark R Goodwin. Artificial intelligence in the control and operation of construction plant-the autonomous robot excavator. Automation in construction, 2(3):217-228, 1993.

[30] Hiroshi Yamamoto, Masaharu Moteki, Hui Shao, Takashi Ootuki, Humihiko Kanazawa, and Yoichi Tanaka. Basic technology toward autonomous hydraulic excavator. In 26th International Symposium on Automation and Robotics in Construction (ISARC 2009), pages 288-295. Citeseer, 2009.

[31] Nathan Melenbrink, Justin Werfel, and Achim Menges. On-site autonomous construction robots: Towards unsupervised building. Automation in Construction, 119:103312, 2020.

[32] Jake Rankin, Laura Justham, Yee Goh, and James Morley. Task delegation and architecture for autonomous excavators. UKRAS20 Conference: "Robots into the real world" Proceedings, 2020. doi:10.31256/ew3zn4z 